EDUardo CAmelo de CAstro'

Rodopiano de SOUZA FLORÊNCIO ${ }^{2}$

Gercino Monteiro Filho ${ }^{3}$

Waldemar NAVES Do AMARAL ${ }^{2}$

\title{
Correlação entre a idade e a contagem dos folículos antrais em mulheres inférteis
}

\section{Correlation between age and antral follicles count in infertile women}

Artigo Original

\begin{abstract}
Palavras-chave
Hormônio folículo estimulante

Folículo ovariano

Ultrassonografia Infertilidade feminina Nomogramas Fatores etários

Keywords

Follicle stimulating hormone Ovarian follicle Ultrasonography Infertility, female

Nomograms Age factors
\end{abstract}

\section{Resumo}

OBJETIVO: Construir um nomograma correlacionando a idade com diferentes valores dos percentis da contagem dos folículos antrais (CFA) em mulheres inférteis. MÉTODOS: Foi feito um estudo transversal de todas as pacientes atendidas, no centro de reprodução assistida Fêmina, no período de $1^{\circ}$ de março de 2010 a $1^{\circ}$ de outubro de 2011 . As pacientes foram submetidas à ultrassonografia transvaginal do $2^{\circ}$ ao $4^{\circ}$ dias de seu ciclo menstrual. Foram incluídas as pacientes de 21 a 45 anos, com ciclos regulares, dois ovários íntegros, sem evidência de endocrinopatias e que assinaram o consentimento. Foram excluídas as tabagistas, portadoras de galactosemia, cistos ovarianos, com antecedente de hepatopatia, cirurgia ginecológico-ovariana e tratamento com quimioterapia ou radioterapia. Com o intuito de se verificar a correlação da CFA com a idade das pacientes, foram utilizados os percentis 5, 25, 50, 75 e 95. Com o uso dos percentis foi feita uma regressão linear que possibilitasse perceber o efeito da idade sobre a CFA. Foi utilizado como nível de significância o valor de 5\% $(p<0,05)$. RESULTADOS: Cento e setenta e duas pacientes foram incluídas no estudo e a média de idade foi de 32,7 anos. Dentre as causas de infertilidade, os fatores masculino e tubário foram as principais etiologias, contribuindo com 65\% dos casos. O nomograma correlacionando a idade com os percentis 5, 25, 50, 75 e 95 da CFA foi melhor ajustado por uma função linear. Os percentis que apresentaram as correlações mais altas foram o P25 $(r=-0,9 ; p<0,001)$, o P50 ( $r=-0,9 ; p<0,001)$ e o P75 (r=-0,9; $p<0,001)$. CONCLUSÃO: Construiu-se um nomograma correlacionando a idade com os diferentes valores dos percentis da CFA em mulheres inférteis sem endocrinopatias. Esse apresentou um padrão linear de redução da CFA com a idade, em todos os percentis. Esse nomograma pode ser uma referência para o clínico; no entanto, uma validação futura, com dados longitudinais, ainda é necessária.

\section{Abstract}

PURPOSE: To produce age-related nomograms for ovarian antral follicle count (AFC) in infertile women. METHODS: It was done a cross-sectional study of patients attended in the center of assisted reproduction Fêmina, from March 2010 to October 2011 . The patients were submitted to transvaginal ultrasonography from day 2 to day 4 of their menstrual period. Patients included were between 21 to 45 years old, with regular menses, two healthy ovaries, without any evidence of endocrinopathies and who gave written informed consent. Patients excluded were smokers, with galactosemia or ovarian cysts, with antecedents of liver disease, ovarian surgeries or who were treated with chemotherapy or radiotherapy. In order to check the evolution of the AFC in relation to patient age, we used the $5^{\text {th }}, 25^{\text {th }}, 50^{\text {th }}, 75^{\text {th }}$ and $95^{\text {th }}$ percentiles. Linear regression was carried out using these percentiles, permitting us to determine the effect of age on the CFA. RESULTS: A total of 172 patients with a mean age of 32.7 years were included in the trial. The male and tubal factors were the main causes of infertility, accounting for $65 \%$ of cases. The age-related nomogram for the $5^{\text {th }}$, $25^{\text {th }}, 50^{\text {th }}, 75^{\text {th }}$ and $95^{\text {th }}$ percentiles of AFC revealed that changes were best fitted by a linear function. The percentiles that showed the highest correlations were 25 ( $r=-0.9 ; p<0.001), 50$ (r=-0.9; $p<0.001)$ and 75 (r=-0.9; $p<0.001)$. CONCLUSION: A nomogram was constructed correlating age with the different AFC percentiles in infertile women without endocrinopathies. This showed a linear pattern of decline in AFC with age in all percentiles. These nomograms could provide a reference guide for the clinician. However, future validation, with longitudinal data, still is needed.
Correspondência

Rodopiano de Souza Florêncio Primeira Avenida $\mathrm{s} / \mathrm{n}$ - Setor Universitário CEP: 74605-020 Goiânia (G0), Brasil

Recebido $22 / 11 / 2011$

' Centro de Reprodução Assistida Fêmina - Humana Medicina Reprodutiva - Goiânia (GO), Brasil.

2 Departamento de Ginecologia e Obstetrícia da Faculdade de Medicina da Universidade Federal de Goiás - UFG - Goiânia (GO),

Brasil.

${ }^{3}$ Faculdade de Medicina da Universidade Federal de Goiás - UFG - Goiânia (GO), Brasil.

Conflito de interesses: não há. 


\section{Introdução}

A reserva ovariana é o pool de folículos ovarianos disponíveis para recrutamento. Representa o potencial funcional do ovário por meio da quantidade e da qualidade dos oócitos ${ }^{1}$. A diminuição da reserva ovariana é um processo de declínio da fecundidade associado tanto à depleção folicular dos ovários, quanto à piora da qualidade oocitária ${ }^{2}$. Provavelmente, há um consumo folicular mais acelerado entre as tabagistas ${ }^{3,4} \mathrm{e}$ em pacientes submetidas a cirurgias ginecológico-ovarianas ${ }^{5}$. O uso de quimioterápicos pode destruir um número importante de folículos primordiais ovarianos ${ }^{6}$. A endometriose pode, também, reduzir artificialmente a contagem de folículos antrais $(\mathrm{CFA})^{7}$.

A reserva ovariana pode variar de forma significativa entre mulheres de mesma idade ${ }^{8}$. Sugere-se, portanto, que apenas a idade não prediga, de forma confiável, a capacidade reprodutiva9. Por isso, métodos clínicos que avaliam essa função têm sido estudados. Destacam-se, entre eles, a $\mathrm{CFA}^{10}$ e os níveis de hormônio antimüleriano $(\mathrm{HAM})^{11}$.

A correlação entre a CFA histológica e o número de folículos primordiais remanescentes, nos ovários das pacientes, tem sido constatada há vários anos ${ }^{7,12,13}$. Nesses anos, vários estudos verificaram, também, uma diminuição no número de folículos primordiais e antrais com o aumento da idade ${ }^{14}$; mas, somente com o aperfeiçoamento dos aparelhos de ultrassonografia, foi possível mostrar a correlação entre a CFA pela ultrassonografia e a idade ${ }^{15,16}$. Desde 2002, vem mostrando-se uma forte correlação entre a CFA menores e a CFA maiores ${ }^{17}$. Por isso, mesmo que tenha sido encontrada uma correlação maior da idade com os folículos antrais com diâmetro de 2 a $6 \mathrm{~mm}$ do que com a CFA total ${ }^{18}$, a padronização de recomendações práticas para a realização desse exame é a contagem dos folículos com diâmetro menor do que $10 \mathrm{~mm}^{7}$. A CFA tem sido avaliada também como preditora de resposta para os tratamentos com indução da ovulação ${ }^{4}$, podendo ter correlação com a quantidade de ciclos cancelados nas más respondedoras ${ }^{9}$, com o número de oócitos aspirados e com a probabilidade de gravidez química ${ }^{19}$. Vários estudos demonstraram a reprodutibilidade das CFA. A CFA é adequada quando feita em duas oportunidades pelo mesmo observador ou por dois observadores diferentes. A CFA apresentou nítida correlação positiva com as concentrações do $\mathrm{HAM}^{20,21}$ e esses foram os dois parâmetros de maior poder preditivo de sucesso no tratamento de reprodução assistida ${ }^{22,23}$. Uma metanálise, com 11 estudos, concluiu que a combinação de múltiplas variáveis (HAM, inibina B, hormônio folículoestimulante (FSH), CFA e outras), para diagnóstico de má respondedora, é comparável ao
CFA isoladamente. Assim, se a opção for por um único exame, esse seria a CFA ${ }^{24}$. O baixo custo da avaliação com a CFA ${ }^{25}$ a necessidade de estabelecimento de nomogramas que correlacionem marcadores da reserva ovariana com a idade ${ }^{26}$ e a presença de estudos sugerindo diferenças étnicas nas curvas de declínio da CFA com a idade ${ }^{27}$ justificam a realização deste estudo em uma população brasileira.

O objetivo deste estudo foi construir um nomograma correlacionando a idade com diferentes valores dos percentis da CFA, em uma população brasileira de pacientes inférteis.

\section{Métodos}

Foi realizado um estudo clínico transversal de todas as pacientes atendidas, pelos autores, no Centro de Reprodução Assistida Fêmina (Goiânia, GO), no período de $1^{\circ}$ de março de 2010 a $1^{\circ}$ de outubro de 2011 . Este estudo foi aprovado pelo Departamento de Ginecologia e Obstetrícia da Faculdade de Medicina da Universidade Federal de Goiás (UFG) e pelo Comitê de Ética em Pesquisa da UFG.

As pacientes foram submetidas à ultrassonografia transvaginal na fase folicular precoce, do $2^{\circ}$ ao $4^{\circ}$ dias do ciclo menstrual. As ultrassonografias foram realizadas apenas pelos médicos pesquisadores, com equipamento Voluson da marca GE com transdutores transvaginais de 7,5 MHz. Todas as medidas dos folículos antrais foram feitas no modo bidimensional. O diâmetro máximo longitudinal e o diâmetro perpendicular foram obtidos com o modo convencional 2D. O tamanho dos folículos foi considerado como a média dessas duas medidas. Os folículos menores ou iguais a $10 \mathrm{~mm}$ de diâmetro foram considerados antrais. Foram avaliados os ovários direito e esquerdo e realizada a somatória das duas contagens. O número total dos folículos antrais foi utilizado para o cálculo. Essas medidas já são feitas de modo rotineiro para avaliação da reserva ovariana da mulher.

O protocolo foi preenchido com a idade da paciente, causa da infertilidade, a contagem ultrassonográfica dos folículos antrais ovarianos, histórico de endocrinopatias, tabagismo, galactosemia e presença de cistos ovarianos durante a CFA. Nesse momento, também foram avaliados os critérios de inclusão e exclusão discriminados abaixo.

Foram incluídas as pacientes com idade entre $21 \mathrm{e}$ 45 anos, com ciclos regulares de 21 a 35 dias, com dois ovários íntegros, sem evidência de doenças endócrinas e que assinaram o informe de consentimento. Foram excluídas as pacientes tabagistas, portadoras de galactosemia, com antecedente de cirurgias ginecológico-ovarianas, tratamento com quimioterapia ou radioterapia, portadoras de cistos 
ou folículos ovarianos medindo mais do que $10 \mathrm{~mm}$ ou com insuficiência hepática.

O programa Microsoft ${ }^{\circledR}$ Excel 2007 foi usado para tabulação dos dados e a análise estatística foi realizada pelo programa Statistical Package for Social Sciences (SPSS ${ }^{\circledR}$ ) for Windows ${ }^{\circledR}$, versão 15.0. Com o intuito de se verificar a correlação da CFA com a idade das pacientes, foram utilizados os percentis 5, 25, 50, 75 e 95. Através desses percentis, foi feita uma regressão linear que possibilitasse perceber o efeito da idade sobre a CFA. Foi utilizado como nível de significância o valor de $5 \%(\mathrm{p}<0,05)$.

\section{Resultados}

Foram incluídas 172 pacientes no estudo e a média de idade foi de 32,7 anos. Dentre as causas de infertilidade, os fatores masculino e tubário foram as principais etiologias, contribuindo com $65 \%$ dos casos. O fator uterino, esterilidade sem causa aparente e síndrome de anticorpo antifosfolipídio também compuseram a casuística.

Setenta e seis por cento $(76 \%)$ das pacientes eram nulíparas, $19 \%$ já tinham tido partos anteriores e $4 \%$ tinham antecedentes de um ou dois abortamentos.

O melhor modelo matemático encontrado para expressar o comportamento da CFA, em relação à idade, em todos os percentis, foi a regressão linear. Observou-se significativa associação entre as variáveis estudadas, isso permitiu estabelecer um modelo de curva de referência a partir dos percentis 5, 25, 50, 75 e 95. Foi realizado um agrupamento das amostras nas faixas etárias mais baixas e mais altas.

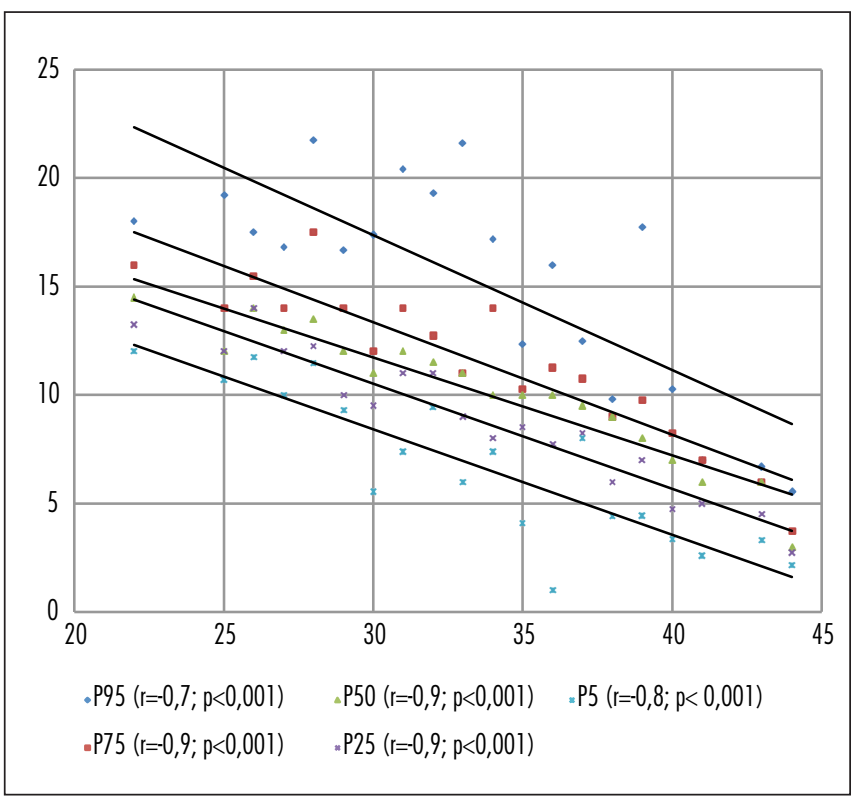

Figura 1. Correlação dos percentis 5, 25, 50, 75, 95 da contagem de folículos antrais com a idade de pacientes inférteis.
A Figura 1 mostra a correlação dos percentis da CFA com a idade. Em todos os percentis, a CFA apresentou uma redução constante com o aumento da idade. A análise de regressão mostrou que, em todos os percentis, houve correlação linear, negativa e forte entre a CFA e a idade das pacientes. Os percentis que apresentaram as correlações mais altas foram o $25(\mathrm{r}=-0,9 ; \mathrm{p}<0,001)$, o $50(\mathrm{r}=-0,9$; $\mathrm{p}<0,001)$ e $075(\mathrm{r}=-0,9 ; \mathrm{p}<0,001)$. A relação entre a idade e a AFC não foi significativamente melhorada por uma análise de regressão não linear.

\section{Discussão}

Nota-se, nos resultados, a predominância dos fatores masculino e tubário de infertilidade. Isso ocorreu por que houve a preocupação em excluir as pacientes com causas não fisiológicas de alteração da reserva ovariana. Muitos estudos disponíveis não excluem mulheres com probabilidade maior de consumo folicular acelerado e incluem tabagistas, portadoras de galactosemia e aquelas que foram submetidas a cirurgias ginecológico-ovarianas ${ }^{28}$.

Um padrão bifásico de redução da CFA com a idade foi descrito em populações de mulheres saudáveis na Holanda $^{29}$. Um estudo de 2004 verificou um padrão linear de diminuição da CFA até os 43 anos $^{30}$. O padrão de queda linear convencional da CFA foi confirmado em vários outros estudos e populações que investigaram a correlação da CFA com a idade ${ }^{10,27,31-33}$.

No ano de 2011, foram publicados os primeiros nomogramas que correlacionaram a CFA com a idade. Um dos estudos foi realizado na Itália, com desenho transversal, e incluiu 362 pacientes saudáveis com ciclos menstruais regulares. Este trabalho excluiu as mulheres com fatores de risco para diminuição acelerada da reserva ovariana. Os autores encontraram uma correlação linear e negativa entre a CFA e a idade em todos os percentis ${ }^{26}$. Esses resultados confirmam o nomograma estabelecido pela casuística deste trabalho brasileiro. Outro trabalho canadense, com 1.880 mulheres inférteis e sem ovário policístico, observou uma correlação linear e alta para o percentil 50 e uma correlação alta e bifásica para os outros percentis. $\mathrm{O}$ estudo discute a possibilidade de que as curvas encontradas apenas representem uma distribuição normal da diminuição da CFA ${ }^{16}$.

Esses nomogramas contribuem, de forma significativa, com a avaliação do efeito da idade na CFA. A identificação de uma paciente jovem com resultado abaixo dos percentis inferiores pode sugerir um sinal de alerta para a sua fertilidade futura. A mudança de percentil, de uma mesma paciente, em duas contagens feitas, em dois momentos distintos e distantes, também pode ter um significado 
clínico. Essa informação pode ser o primeiro passo para o manejo e aconselhamento das pacientes.

Outros nomogramas comumente utilizados, tais como as curvas de crescimento fetal e de peso ao nascer são baseadas em dados transversais. A despeito disso, estudos longitudinais são necessários para validar todos esses nomogramas construídos por meio de estudos transversais.

Este estudo foi realizado com pacientes inférteis e isso pode limitar a aplicação do nomograma para uma população de pacientes férteis. Outra cautela que deve ser observada é que o nomograma foi construído com paciente que apresentavam ciclos regulares e dois ovários íntegros. Também, foram excluídas as pacientes com fatores de risco para diminuição acelerada da reserva ovariana e as portadoras de endocrinopatias.

Concluímos que o nomograma correlacionando a idade com os diferentes valores dos percentis da CFA em mulheres inférteis sem endocrinopatias apresentou um padrão linear de redução da CFA com a idade, em todos os percentis. Esse nomograma pode ser uma referência para o clínico; no entanto, uma validação futura com dados longitudinais ainda é necessária.

\section{Referências}

1. Maheshwari A, Fowler P, Bhattacharya S. Assessment of ovarian reserve - should we perform tests of ovarian reserve routinely? Hum Reprod. 2006;21(1 1):2729-35.

2. Broekmans FJ, Kwee J, Hendriks DJ, Mol BW, Lambalk CB. A systematic review of tests predicting ovarian reserve and IVF outcome. Hum Reprod Update. 2006;12(6):685-718.

3. El-Nemr A, Al-Shawaf T, Sabatini L, Wilson C, Lower AM, Grudzinskas JG. Effect of smoking on ovarian reserve and ovarian stimulation in in-vitro fertilization and embryo transfer. Hum Reprod. 1998; 13(8):2192-8.

4. Popovic-Todorovic B, Loft A, Lindhard A, Bangsbøll S, Andersson $A M$, Andersen AN. A prospective study of predictive factors of ovarian response in standard IVF/ICSI patients treated with recombinant $\mathrm{FSH}$. A suggestion for a recombinant $\mathrm{FSH}$ dosage normogram. Hum Reprod. 2003; 18(4):781-7.

5. Melica F, Chiodi S, Cristoforoni PM, Ravera GB. Reductive surgery and ovarian function in the human: can reductive ovarian surgery in reproductive age negatively influence fertility and age at onset of menopause? Int J Fertil Menopausal Stud. 1995;40(2):79-85.

6. Wallace WH, Kelsey TW. Ovarian reserve and reproductive age may be determined from measurement of ovarian volume by transvaginal sonography. Hum Reprod. 2004;19(7):1612-7.

7. Broekmans FJ, de Ziegler D, Howles CM, Gougeon A, Trew G, Olivennes $F$. The antral follicle count: practical recommendations for better standardization. Fertil Steril. 2010;94(3):1044-51.

8. Burger HG, Dudley EC, Robertson DM, Dennerstein L. Hormonal changes in the menopause transition. Recent Prog Horm Res. 2002;57(1):257-75.

9. Jain T, Soules MR, Collins JA. Comparison of basal follicle-stimulating hormone versus the clomiphene citrate challenge test of ovarian reserve screening. Fertil Steril. 2004;82(1):180-5.

10. Rosen MP, Sternfeld B, Schuh-Huerta SM, Reijo Pera RA, McCulloch $\mathrm{CE}$, Cedars MI. Antral follicle count: absence of significant midlife decline. Fertil Steril. 2010;94(6):2182-5.

11. Almog B, Shehata F, Suissa S, Holzer H, Shalom-Paz E, La Marca $A$, et al. Age-related normograms of serum antimüllerian hormone levels in a population of infertile women: a multicenter study. Fertil Steril. 2011 ;95(7):2359-63.

12. Block E. Quantitative morphological investigations of the follicular system in women; variations at different ages. Acta Anat (Basel). $1952 ; 14(1-2): 108-23$.
13. Gougeon A, Chainy GB. Morphometric studies of small follicles in ovaries of women at different ages. J Reprod Fertil. 1987;81(2):433-42.

14. Faddy M, Gosden RG, Gougeon A, Richardson SJ, Nelson JF. Accelerated disappearance of ovarian follicles in mid-life: implications for forecasting menopause. Hum Reprod. 1992;7(10):1342-6.

15. Reuss ML, Kline J, Santos R, Levin B, Timor-Tritsch I. Age and the ovarian follicle pool assessed with transvaginal ultrasonography. Am J Obstet Gynecol. 1996;174(2):624-7.

16. Almog B, Shehata F, Shalom-Paz E, Tan SL, Tulandi T. Age-related normogram for antral follicle count: McGill reference guide. Fertil Steril. 2011;95(2):663-6.

17. Bancsi LF, Broekmans FJ, Eijkemans M, de Jong FH, Habbema $J D$, te Velde ER. Predictors of poor ovarian response in in vitro fertilization: a prospective study comparing basal markers of ovarian reserve. Fertil Steril. 2002;77(2):328-36.

18. Haadsma ML, Bukman A, Groen H, Roeloffzen EM, Groenewoud ER, Heineman $M$, et al. The number of small antral follicles (2-6 $\mathrm{mm}$ ) determines the outcome of endrocrine ovarian reserve tests in a subfertile population. Hum Reprod. 2007;22(7):1925-31.

19. Muttukrishna S, McGarrigle H, Wakim R, Khadum I, Ranieri DM, Serhal P. Antral follicle count, anti-mullerian hormone and inhibin $B$ : predictors of ovarian response in assisted reproductive technology? BJOG. 2005; 1 12(10): 1384-90.

20. Fanchin R, Schonäuer LM, Righini C, Guibourdenche J, Frydman $R$, Taieb J. Serum anti-Müllerian hormone is more strongly related to ovarian follicular status than serum inhibin $B$, estradiol, FSH and LH on day 3. Hum Reprod. 2003;18(2):323-7.

21. Visser JA, Themmen AP. Anti-Müllerian hormone and folliculogenesis. Mol Cel Endocrinol. 2005;234(1-2):81-6.

22. Jayaprakasan K, Hilwah N, Kendall NR, Hopkisson JF, Campbell $B K$, Johnson IR, et al. Does 3D ultrasound offer any advantage in the pretreatment assessment of ovarian reserve and prediction of outcome after assisted reproduction treatment? Hum Reprod. 2007;22(7): 1932-41.

23. Scheffer GJ, Broekmans FJ, Bancsi LF, Habbema JD, Looman CW, Te Velde ER. Quantitative transvaginal two-and three-dimensional sonography of the ovaries: reproducibility of antral follicle counts. Ultrasound Obstet Gynecol. 2002;20(3):270-5.

24. Verhagen TE, Hendriks DJ, Bancsi LF, Mol BW, Broekmans FJ. The accuracy of multivariate models predicting ovarian reserve and 
pregnancy after in vitro fertilization: a meta-analysis. Hum Reprod Update. 2008; 14(2):95-100.

25. Castro EC, Amaral WN. Avaliação da reserva ovariana. Reprod Clim. 2009;24(3):1-8.

26. La Marca A, Spada E, Sighinolfi G, Argento C, Tirelli A, Giulini $S$, et al. Age -specific nomogram for the decline in antral follicle count throughout the reproductive period. Fertil Steril. $2011 ; 95(2): 684-8$.

27. Ng EH, Yeung WS, Fong DY, Ho PC. Effects of age on hormonal and ultrasound markers of ovarian reserve in Chinese women with proven fertility. Hum Reprod. 2003;18(10):2169-74.

28. de Medeiros SF, Assi PE, de Medeiros MM. Gonadotrophin dynamics during reproductive life. Int J Gynaecol Obstet. 2004;87(1):24-8.

29. Scheffer GJ, Broekmans FJ, Dorland M, Habbema JD, Looman CW, te Velde ER. Antral follicle counts by transvaginal ultrasonography are related to age in women with proven natural fertility. Fertil Steril. 1999;72(5):845-51.

30. Broekmans FJ, Faddy M, Scheffer G, te Velde ER. Antral follicle counts are related to age at natural fertility loss and age at menopause. Menopause. 2004; 11 (6 Pt 1):607-14.

31. Tufan E, Elter K, Durmusoglu F. Assessment of reproductive ageing patterns by hormonal and ultrasonographic ovarian reserve tests. Hum Reprod. 2004;19(1 1):2484-9.

32. Pastor CL, Vanderhoof VH, Lim LC, Calis KA, Premkumar A, Guerrero NT, et al. Pilot study investigating the age-related decline in ovarian function of regularly menstruating normal women. Fertil Steril. 2005;84(5): 1462-9.

33. Al-Sunaidi M, Al-Mahrizi S, Tan SL, Tulandi T. Age-related changes in antral follicle count among women with and without polycystic ovaries. Gynecol Obstet Invest. 2007;64(4):199-203. 